\title{
Expansion and Contraction Modulate Visual Short-Term Memory
}

\author{
Junichi Takahashi', Yousuke Kawachi', and Jiro Gyoba ${ }^{3}$ \\ 1 Faculty of Human Development and Culture, Fukushima University \\ 2 Department of Psychology for Human Well-being, Faculty of General Welfare, Tohoku Fukushi University \\ ${ }^{3}$ Department of Psychology, Shokei Gakuin University
}

ABSTRACT

We examined the influence of expansion and contraction on visual short-term memory (VSTM) using a change-detection task. In each trial, several expanding/contracting line segments with various orientations were presented in two successive displays. The orientation of objects in the second display was either identical to, or different from, that of the first display. Observers were asked to judge the presence or absence of an orientation change in successive displays. Results showed that memory performance for expanding objects was higher than for contracting objects (expansion benefit: Experiments 1A and 1B). Further experiments focused on VSTM processing (encoding, storage, and retrieval). Regarding the retrieval stage, an expansion benefit was replicated only when the direction of motion was consistent between two successive displays (Experiment 2A). A cueing stimulus enhanced the memory performance for both expanding and contracting motions and eliminated the expansion benefit (Experiment 2B). Regarding the storage stage, we found the expansion benefit occurred only for shorter blank intervals between the two displays (Experiment 3). Regarding the encoding stage, the expansion benefit was observed regardless of presentation times (Experiment 4). These results indicate the possibility that expanding and contracting motions modulate VSTM.

\section{INTRODUCTION}

To adapt to its current environment, the visual system needs to gather sensory information about its characteristics and create perceptual representations, which contribute to decision making (e.g., Sheth \& Pham, 2008). However, the visual system has limited capacity and thus it has to effectively choose among the available perceptual information to create meaningful representations. This process is supported by visual short-term memory (VSTM).

An early component of visual memory processes, VSTM encodes and temporary stores object representations. Researchers have exam- ined two topics related to VSTM (e.g., Jiang, Olson, \& Chun, 2000): its capacity and representation. Regarding the former, many researchers (Alvarez \& Cavanagh, 2004; Luck \& Vogel, 1997; Phillips, 1974) have shown that VSTM's capacity may be limited to approximately five objects. The latter concerns what factors can be represented in VSTM, which has been examined with various experimental stimuli, such Corresponding author: Junichi Takahashi, Faculty of Human Development and Culture, Fukushima University, 1 Kanayagawa, Fukushima-shi, Fukushima 9601296, Japan. e-mail: j-takahashi@educ.fukushima-u.ac.jp 
as Gabor patches, random polygons, faces, and spatial configuration between objects (Alvarez \& Cavanagh, 2004, 2008; Fougnie, Suchow, \& Alvarez, 2012; Franconeri, Alvarez, \& Cavanagh, 2013; Jiang et al., 2000; Jiang, Shim, \& Makovski, 2008). Recently, the effects of threatening objects and affective properties on VSTM representation have been found by using stimuli such as natural scenes, pictures, visual patterns, and emotional faces (e.g., Jackson \& Raymond, 2008; Jackson, Wolf, Johnston, Raymond, \& Linden, 2008; Jackson, Wu, Linden, \& Raymond, 2009; Mather et al., 2006; Öhman, Flykt, \& Esteves, 2001; Takahashi, Kawachi, \& Gyoba, 2015).

Regarding threatening objects from an ecological viewpoint, the eye has an important ability to detect objects that are moving toward it. It enables the viewer to avoid threatening environmental situations (Shirai et al., 2009; Shirai, Kanazawa, \& Yamaguchi, 2004, 2006, 2008; Takeuchi, 1997). To examine this ability, researchers have used expanding and contracting motions to create the impression of motion toward or away from the eye, respectively. For example, Takeuchi examined the effect of expanding and contracting motions on visual search efficiency. The results revealed a search asymmetry between expanding and contracting objects. The search time needed to find an expanding target did not vary depending on the number of distractors, which were defined by contracting objects. In contrast, when searching for a contracting target among expanding distractors, the visual system's search time increased with the number of distractors. Thus, this study proposes that the visual system has a differential sensitivity, preferentially detecting expanding over contracting motion.

Since the visual system is more likely to create meaningful, effective representations more quickly when objects imply more threatening environmental situations (Öhman et al., 2001), we assumed that VSTM processing would support memory for expanding and contracting motions. Although several studies have examined the effect of motion information, such as the speed or temporal frequency of moving random dots on VSTM (e.g., McKeefry, Burton, \& Vakrou, 2007; Pasternak \& Zaksas, 2003; Zaksas, Bisley, \& Pasternak, 2001), few reports have investigated the modulation of expanding and contracting motions on VSTM. Previous studies (Franconeri \& Simons, 2003; Kawahara, Yanase, \& Kitazaki, 2012) have examined how expanding and contracting motions capture visual attention. Kawahara et al. (2012) used expansion, contraction, and static conditions as experimental stimuli (optical flow) and showed that the expansion condition more dominantly captured visual attention compared with the contraction condition. The present study adds a potential new modulator to VSTM representation. Namely, we determined whether expanding and contracting motions modulate VSTM's encoding, storage, and retrieval stages in addition to attention capturing.

In the present study, we examined the effects of expanding and contracting motion on VSTM. Specifically, we examined whether representations of expanding and contracting motions had equal effects on the encoding, storage, and retrieval stages of VSTM processing. Since there is higher sensitivity to expanding motion in terms of visual detection (e.g., Takeuchi, 1997), we hypothesized that expanding motion will be preferentially registered in VSTM representation, in contrast to contracting motion, indicating that memory performance for expanding objects might be larger than that for contracting objects.

\section{GENERAL METHOD}

\section{Participants}

All participants (aged 19-35 years) were either undergraduate or graduate students. Twelve (three men and nine women: Experiment $1 \mathrm{~A}$ ), seven (five men and two women: Experiment 1B), nine (two men and seven women: Experiment 2A), seven (two men and five women: Experiment 2B), 14 people (five men and nine women: Experiment 3), and 11 people (two men and nine women: Experiment 4) participated in each experiment. All participants had normal or corrected-to-normal visual acuity and provided informed consent before participating.

\section{Apparatus}

The presentations of stimuli and data collection were controlled by PC: a Panasonic CF-Y5 in Experiment 1A and a Dell Precision 390 in Experiments $1 \mathrm{~B}$ to 4 . All the stimuli were generated using MATLAB (Math Works Inc.) and Cogent Graphics packages. In Experiment 1A, stimuli were displayed on a Mitsubishi Diamondtron M2 RDF223H, 22 in. CRT monitor) and in Experiments 1B to 4, on a Sony Trinitron GDM-FW900, SONY, 24 in. CRT monitor. For both PCs and displays, a $60 \mathrm{~Hz}$ refresh rate, controlled luminance of stimuli $\left(64.0 \mathrm{~cd} / \mathrm{m}^{2}\right)$, and background $\left(0.5 \mathrm{~cd} / \mathrm{m}^{2}\right)$ were used.

\section{Stimuli}

Figure 1 shows the stimuli used in this study. The white line segments were set at either 0,45 , or 135 degrees of orientation $\left(64.0 \mathrm{~cd} / \mathrm{m}^{2}\right)$ and featured either an expanding motion, from 0.3 (horizontal) $\times 0.7$ (vertical) to $0.9 \times 2.1$ degrees of visual angle, or a contracting motion, from $0.9 \times 2.1$ to $0.3 \times 0.7$ degrees of visual angle. The stimuli were presented in random positions within a $4 \times 4$ virtual grid subtending $9 \times 9$ degrees of visual angle on a black background $\left(0.5 \mathrm{~cd} / \mathrm{m}^{2}\right)$.

\section{Procedure}

We measured memory performance (accuracy) by using a modified change-detection task based on Jiang, et al. (2000, see Figure 1, Panel C). A participant sat in a seat approximately $60 \mathrm{~cm}$ away from the CRT monitor with their head resting on a chinrest. On the monitor, line segments with an expanding (or contracting) motion were presented for $667 \mathrm{~ms}$ per trial (the memory display), followed by a presentation of the mask stimulus for $100 \mathrm{~ms}$. After a blank interval of $400 \mathrm{~ms}$, the test display with an expanding (or contracting) motion was presented for $667 \mathrm{~ms}$ in a fashion similar to the memory display. The test display was either identical to the memory display or contained a line segment with a different orientation. Next, a blank display appeared until the participant responded. Participants were asked to judge whether the orientation of the line segment in the memory and test displays was the same or different. Response accuracy rather than speed was emphasized. 
(a)

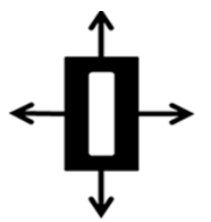

Expanding motion objects Contracting motion objects (b)

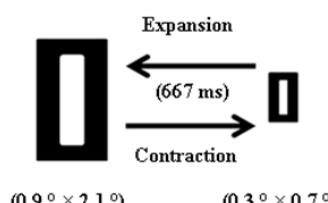

(c)

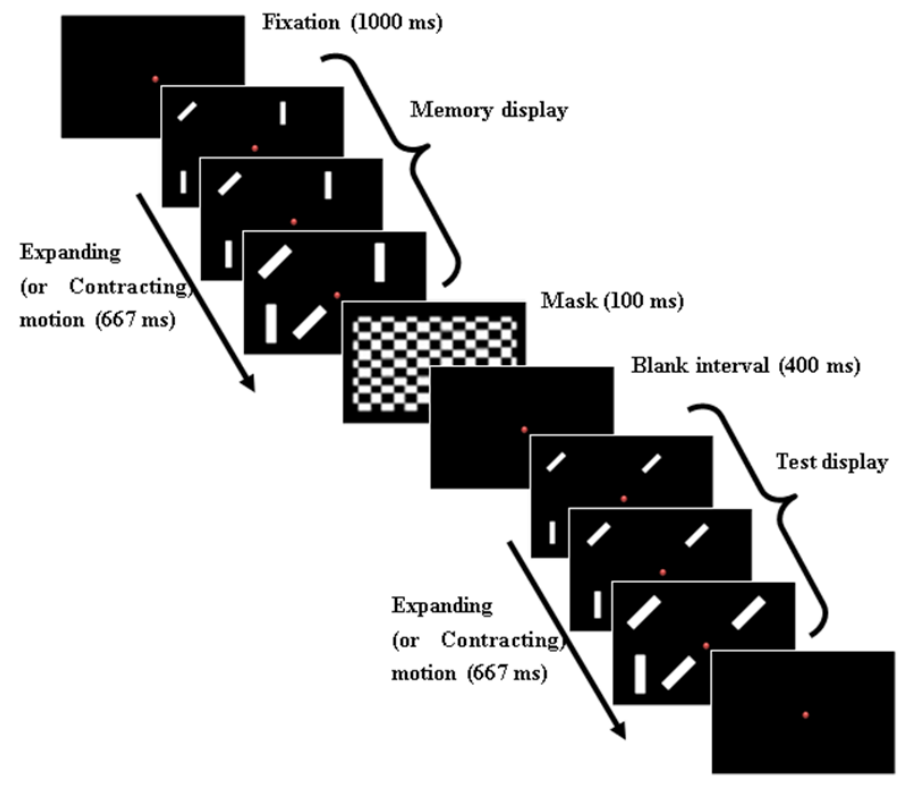

FIGURE 1.

Response

Panel A: Samples of the line segments used in this study. Panel B: The line segments with expanding/contracting motion. The line segments expanded from 0.3 (horizontal) $\times 0.7$ (vertical) to $0.9 \times 2.1$ degrees of visual angle and contracted from $0.9 \times 2.1$ to $0.3 \times 0.7$ degrees of visual angle. Panel C: A stimulus sequence from the present study. In each trial, two displays were presented, separated by a blank interval. The memory display contained several line segments. The second (test) display contained line segments, each of which was identical to that of the first (memory) display or only one of which was changed in orientation. Participants were required to respond whether the two displays were the same or different. This sample sequence represents the different trial in the condition of expanding motion.

\section{EXPERIMENT 1:}

\section{THE MODULATORY EFFECT OF EXPAND- ING AND CONTRACTING MOTIONS ON VISUAL SHORT-TERM MEMORY}

In Experiments $1 \mathrm{~A}$ and $1 \mathrm{~B}$, we report that expanding motion dominantly modulates memory performance compared with contracting motion.

\section{Experiment 1A}

\section{METHOD}

Twelve people (three men and nine women) who were naive to the purpose of the study participated in Experiment 1A. The stimuli were line segments containing expanding or contracting motion, as specified in the General Method section. To compare memory performance between objects with motion (expanding and contracting) and those without, we used static line segments $(0.6 \times 1.4$ degrees of visual angle).
In the change-detection task, the two factors of motion (expansion, contraction, or without motion) and set size $(1,3,5$, or 7$)$ were controlled for. Each participant completed 576 trials ( 3 motions $\times 4$ set sizes $\times 2$ conditions [same or different] $\times 24$ trials) in a random order.

\section{RESULTS AND DISCUSSION}

Figure 2 shows the accuracy of all the participants in Experiment 1A. To examine the results $(n=12)$, a two-way ANOVA was conducted on the factors of motion and set size, and a significant interaction was found, $F(6,66)=6.57, p=.001, \eta_{\mathrm{p}}{ }^{2}=.37$. A simple main effect of motion was observed in Set sizes 5 and $7, F(2,88)=$ $19.66, p=.001, \eta_{\mathrm{p}}{ }^{2}=.31$ and $F(2,88)=27.79, p=.001, \eta_{\mathrm{p}}{ }^{2}=.39$, respectively. The post-hoc analysis revealed that the accuracy for no motion was the best and the accuracy for contraction was the worst in Set size 5 (see Supplementary Table 1). Also, a simple main effect was observed for set size in all motions, $F(3,99)=71.47, p$ $=.001, \eta_{\mathrm{p}}{ }^{2}=.68$ for expansion, $F(3,99)=110.38, p=.001, \eta_{\mathrm{p}}{ }^{2}=$ .77 for contraction, and $F(3,99)=37.98, p=.001, \eta_{\mathrm{p}}{ }^{2}=.54$ for no 
motion. The post-hoc analysis revealed that the accuracy for Set sizes 1 and 3 was significantly better than that for Set sizes 5 and 7 , and the accuracy for Set size 5 was higher than that for Set size 7 in expansion, contraction, and without motion (see Supplementary Table 1). Moreover, the results showed significant main effects of motion, $F(2,22)=23.23, p=.001, \eta_{\mathrm{p}}{ }^{2}=.68$ and set size, $F(3,33)=$ $150.72, p=.001, \eta_{\mathrm{p}}{ }^{2}=.93$. With regard to the main effect of motion, the post-hoc analysis revealed that the accuracy for no motion was the best, while it was the worst for contraction (see Supplementary Table 1). Regarding the main effect of set size, the post-hoc analysis revealed that the accuracy for Set sizes 1 and 3 were significantly better than that for Set sizes 5 and 7, and the accuracy for Set size 5 was higher than that for Set size 7 (see Supplementary Table 1).

Experiment 1A's required sample size was confirmed by calculating the effect size with a power level $(1-\beta)$ of 0.8 (see Cohen, 1992). In the analysis among motions (a simple main effects of motion in Set sizes 5 and 7 revealed by the interaction between motion and set size, $\eta_{\mathrm{p}}{ }^{2}=.31$ and $\eta_{\mathrm{p}}{ }^{2}=.39$ ), we found that a total sample size of 12 was necessary for obtaining results with an $\alpha$ of 0.05 . Thus, we assumed that our sample size was sufficient for Experiment 1A.

Experiment 1A showed that the accuracy for expanding motion was larger than that for contracting motion (the expansion benefit). Since the memory performance for expanding motion was lower than that for no motion (static line segments), the expansion benefit may be limited to the psychological phenomenon that was observed under the motion condition (expansion and contraction) in VSTM.

One might argue that memory performance was modulated by the object size in the last flame of the motion animation, because it was larger for objects that contained expanding motion than for those that contained contracting motion. In Experiment 1B, we addressed this possibility using a similar experimental design as in Experiment 1A, in which the difference was that the last frame depicting both the expanding and contracting motions in the changedetection task were controlled as the same visual angle.

\section{Experiment 1B}

\section{METHOD}

Seven people (five men and two women) participated in Experiment $1 \mathrm{~B}$, including the two authors and five additional people who were naive to the purpose of the study. The only change was that we controlled the objects' size in the last frame of the expanding motion (from $0.3 \times 0.7$ to $0.6 \times 1.4$ degrees of visual angle) and the contracting motion (from $0.9 \times 2.1$ to $0.6 \times 1.4$ degrees of visual angle, see Figure 3, Panel A).

The two factors of motion (expansion or contraction) and set size $(1,3,5$, or 7$)$ were examined. Each participant completed 384 trials $(2$ motions $\times 4$ set sizes $\times 2$ conditions [same or different] $\times$ 24 trials), which were presented in random order.

\section{RESULTS AND DISCUSSION}

Figure 3, Panel B shows the accuracy across all participants in Experiment 1B. We conducted a two-way ANOVA on the factors of motion and set size. The results showed significant main effects of motion, $F(1,6)=11.18, p=.02, \eta_{p}{ }^{2}=.65$ and set size, $F(3,18)$ $=78.55, p=0.001, \eta_{\mathrm{p}}{ }^{2}=.93$. With regard to the main effect of set size, our post-hoc analysis showed that the accuracy for Set sizes 1 and 3 was significantly better than that for Set sizes 5 and 7, and the accuracy for Set size 5 was higher than that for Set size 7 (see Supplementary Table 2). Moreover, a significant interaction between motion and set size was observed, $F(3,18)=6.60, p=.003$, $\eta_{\mathrm{p}}{ }^{2}=.52$. A simple main effect of motion was observed in Set sizes 5 and $7, F(1,24)=15.96, p=.001, \eta_{\mathrm{p}}{ }^{2}=.40$ for Set size 5 and $F(1,24)$ $=15.96, p=.001, \eta_{\mathrm{p}}^{2}=.40$ for Set size 7 , respectively, confirming that the accuracy for expanding motion was higher than that for contracting motion in these set sizes. Also, we observed a simple main effect of set size in expanding and contracting motions, $F(3$, $36)=36.40, p=.001, \eta_{\mathrm{p}}^{2}=.75$ for expanding and $F(3,36)=76.29$,

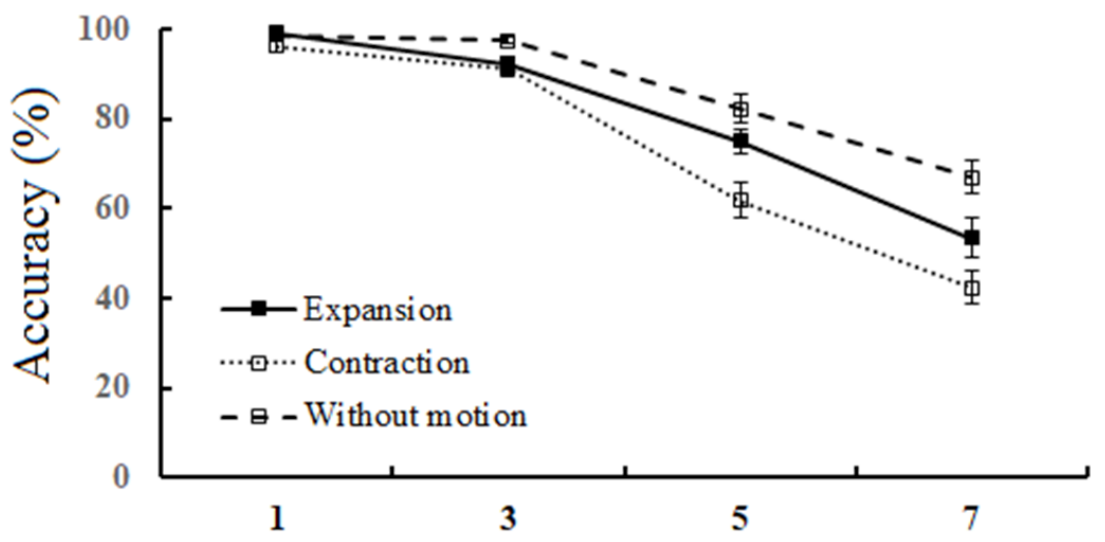

Set size

FIGURE 2.

Mean accuracy of Experiment 1A ( $\boldsymbol{n}=12)$. Error bars denote the standard error of the means. The solid, dotted, and broken lines represent the accuracy for expanding, contracting, and without motion. 
$p=.001, \eta_{\mathrm{p}}{ }^{2}=.86$ for contracting motion. The post-hoc analysis revealed that the accuracy for Set sizes 1 and 3 was significantly better than that for Set sizes 5 and 7, and the accuracy for Set size 5 was higher than that for Set size 7 for expansion and contraction (see Supplementary Table 2).

We calculated the required sample size using an effect size (significant interaction between motion and set size, $\eta_{\mathrm{p}}{ }^{2}=.52$ ) with a power level $(1-\beta)$ of 0.8 . We found that a total sample size of 6 was necessary for obtaining results with an $\alpha$ of 0.05 . Thus, our sample size $(n=7)$ was sufficient to for Experiment $1 \mathrm{~B}$.

Experiment $1 \mathrm{~B}$ revealed that after controlling for the final size of objects, the memory performance for objects with contracting motion was lower than that for expanding motion when set sizes were greater than five. Thus, we can eliminate the possibility that memory performance was modulated by the object size in the last frame of the motion animation. Based on the results of Experiments $1 \mathrm{~A}$ and $1 \mathrm{~B}$, we propose the modulatory effect of expanding motion (i.e., expansion benefit compared with contraction) in VSTM although the memory performance for both motions was lower than for without motion (static line segments).

Visual short-term memory processing is composed of three stages: encoding, storage, and retrieval (Alvarez \& Cavanagh 2004). We examined critical factors of the modulatory effect when the expansion benefit occurs in the later retrieval stage (Experiments $2 \mathrm{~A}$ and $2 \mathrm{~B}$ ), the storage stage (Experiment 3 ), and in the early encoding stage (Experiment 4).

\section{EXPERIMENT 2:}

\section{THE EXPANSION BENEFIT IN THE RE- TRIEVAL STAGE OF VISUAL SHORT-TERM MEMORY}

To examine the expansion benefit in the retrieval stage, Experiment 2 manipulated the contents of the test display in the change-detection task. In Experiment 2A, we examined motion congruency between the memory and test displays, with either: (a) expanding or contracting motion in the memory display and the same motion in the test display (congruous condition); (b) different motions between the memory and test displays (incongruous condition), and (c) expanding or contracting motion in the memory display and static line segments in the test display (static condition). If the expansion benefit was modulated by the contents of the test display, then we would observe it only in the congruous condition, as found in Experiment 1A. If the expansion benefit were not affected by the contents of the test display, however, then we would observe the effect under all conditions.

\section{Experiment 2A}

\section{METHOD}

Nine people (two men and seven women) naive to the study's purpose took part in Experiment 2A (five new participants). The same design as in Experiment 1A was used, with the exception that we manipulated the motion congruency between the memory and test displays (see Figure 4). The direction of motion in the test display was identical to that in the memory display in the congruous condition; in the incongruous condition, the motion was different between the memory and test displays. In addition, for the static condition, only the memory display had an expanding or contracting motion while the test display had static line segments $(0.6 \times 1.4$ degrees of visual angle).

The three factors of motion (expansion or contraction), set size $(1,3,5$, or 7$)$, and motion congruency (congruous, incongruous, or static) were examined. Therefore, each participant completed 1152 trials $(2$ motions $\times 4$ set sizes $\times 3$ motion congruencies $\times 2$ conditions [same or different] $\times 24$ trials) with the order of motion congruency conditions counterbalanced across participants. (a)

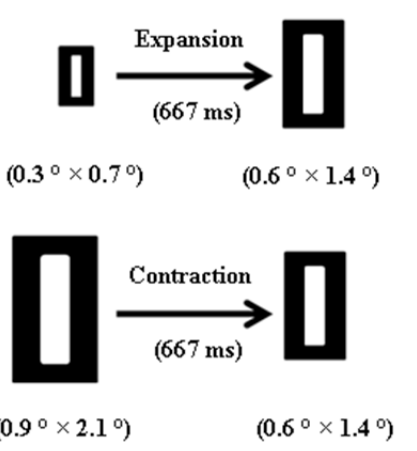

(b)

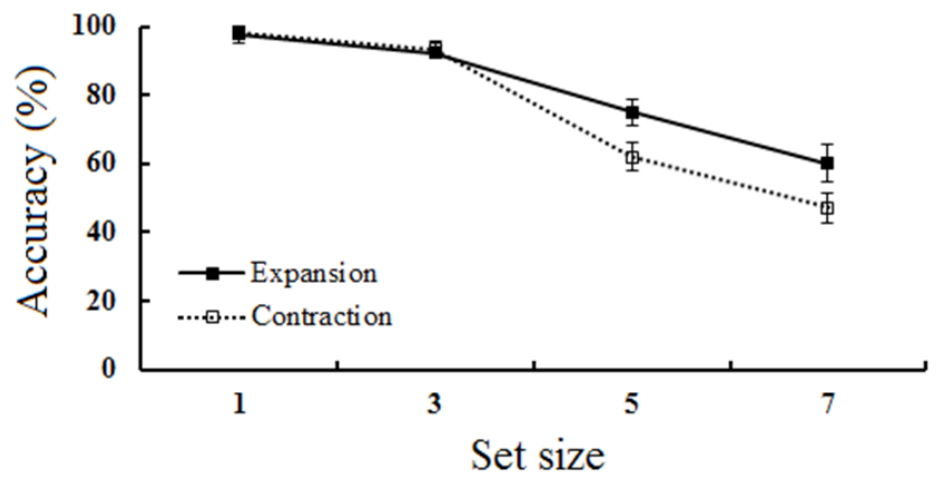

|FIGURE 3.

Panel A: The line segments with expanding/contracting motion used in Experiment 1B. To control the object's size in the last frame of the stimulus, the line segments expanded from 0.3 (horizontal) $\times 0.7$ (vertical) to $0.6 \times 1.4$ degrees of visual angle and contracted from $0.9 \times 2.1$ to $0.6 \times 1.4$ degrees of visual angle. Panel B: Mean accuracy of Experiment $1 \mathrm{~B}(n=7)$. Error bars denote the standard error of the means. The solid line represents the accuracy for expanding motion and the dotted line represents the accuracy for contracting motion. 
(a)

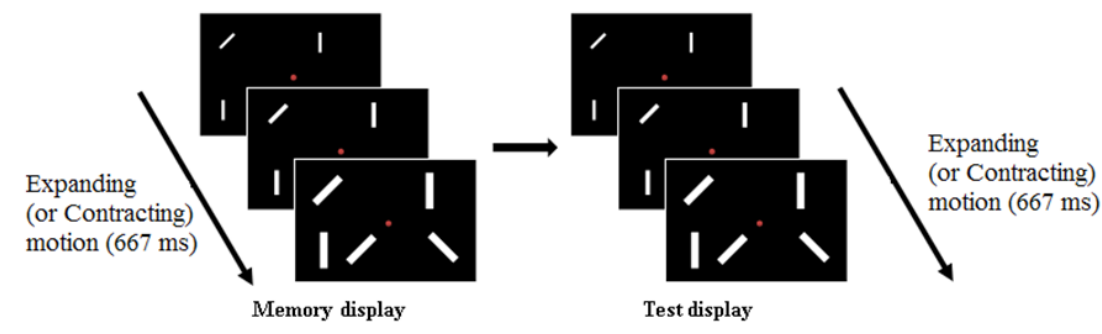

(b)

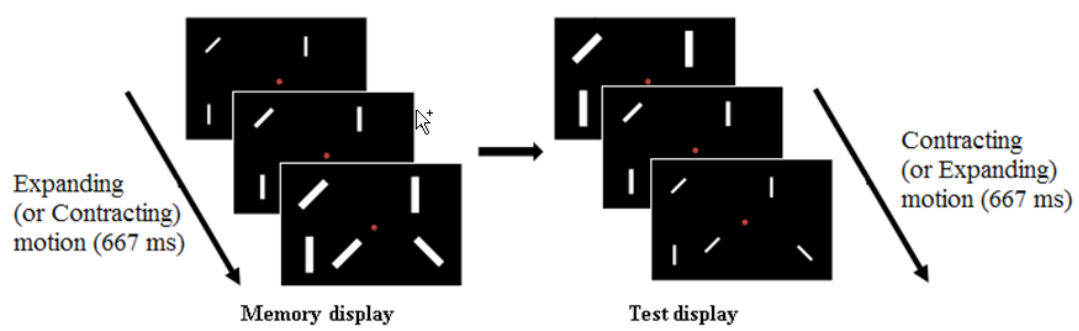

(c)

|FIGURE 4.

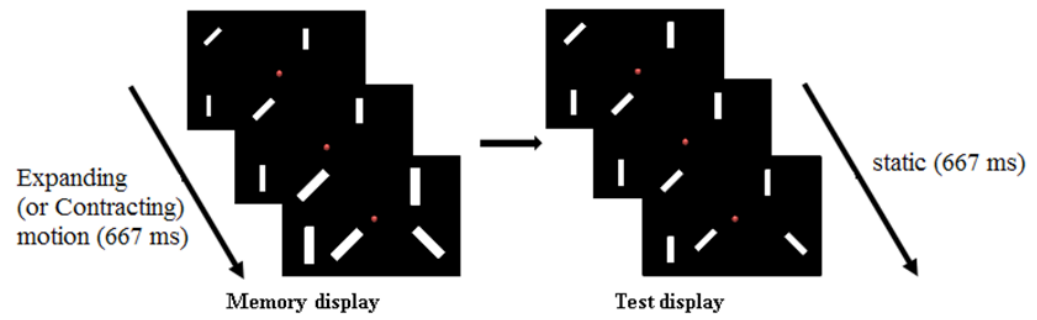

Samples of the motion conditions in Experiment 2A. Panel A: Congruous condition: the motion of line segments in the memory display was congruous with that of the test display. Panel B: Incongruous condition: the motion of line segments in the memory display was incongruous with that of the test display. Panel C: Static condition: the line segments in the memory display contained expanding (or contracting) motion whereas the test display contained static line segments.

\section{RESULTS AND DISCUSSION}

We conducted a three-way ANOVA on the factors of motion, set size, and motion congruency (see Figure 5). There were significant main effects for all three factors, $F(1,8)=9.13, p=.02$, $\eta_{\mathrm{p}}{ }^{2}=.53$ for motion, $F(3,24)=61.27, p=.001, \eta_{\mathrm{p}}{ }^{2}=.88$ for set size, and $F(2,16)=5.38, p=.02, \eta_{\mathrm{p}}{ }^{2}=.40$ for motion congruency. With regard to the main effect of set size, the post-hoc analysis showed that the accuracy for Set sizes 1 and 3 was significantly better than that for Set sizes 5 and 7, and the accuracy for Set size 5 was higher than that for Set size 7 (see Supplementary Table 3). Also, regarding the main effect of motion congruency, the accuracy for the static condition was higher than that for the congruous condition, $t(16)=3.18, p=.006$. Moreover, we observed a significant interaction between motion and motion congruency, $F(2,16)=4.13, p=$ $.04, \eta_{\mathrm{p}}{ }^{2}=.34$. Regarding the significant interaction between motion and motion congruency, a simple main effect of motion congruency was observed in contracting motion, $F(2,32)=7.43, p=.002$, $\eta_{\mathrm{p}}{ }^{2}=.32$. The post-hoc analysis revealed that the accuracy for the static condition was higher than that for the congruous condition for contracting motion, $t(32)=3.85, p=.001$. Also, a simple main effect of motion was observed in the congruous condition, $F(1,24)=16.49, p=.001, \eta_{p}{ }^{2}=0.41$, confirming that the accuracy for the contracting motion was lower than that for the expanding motion in the congruous condition. However, we did not observe interactions between motion congruency and set size, $F(6,48)=1.91, p=.09, \eta_{\mathrm{p}}{ }^{2}=.19$, between motion and set size, $F(3,24)=0.42, p=.74, \eta_{\mathrm{p}}{ }^{2}=.05$, or among these three factors, $F(6,48)=1.21, p=.32, \eta_{\mathrm{p}}{ }^{2}=.13$.

To examine the required sample size, we calculated it using an effect size (significant interaction between motion and motion congruency: $\eta_{p}{ }^{2}=.34$ ) with a power level $(1-\beta)$ of 0.8 , showing that a total sample size of 6 was necessary for obtaining results with an $\alpha$ of 0.05 . We thus assumed that our sample size $(n=9)$ would be sufficient for examining the results of Experiment 2A.

From the results of Experiment 2A, where differences in accuracy were observed only in the congruous condition, we can assume that the expansion benefit can only be observed when the memory and test displays contain the same motion. When the test display contained incongruous motion or static line segments, the expansion benefit was not observed. Also, we found that the memory 
performance for expanding motion was similar, regardless of the contents of the test display.

The contents of the test display affected the occurrence of the expansion benefit, suggesting that differences in memory performance between expanding and contracting motion were observed in the retrieval stage of memory processes. In Experiment 2B, we further confirmed the modulation of expanding and contracting motions in the retrieval stage by using a cueing stimulus in the test display (Wheeler \& Treisman, 2002; Yang, Tseng, \& Wu, 2015). A cueing stimulus enhances the memory performance because the participants have only to compare the cued stimulus between the memory and test displays, reducing the amount of decision load. On the other hand, participants have to compare all stimuli between the displays, increasing the decision load. The modulation is varied depending on the decision load (cueing effect) in the test display, meaning that the modulation occurs in the retrieval stage,

(a)

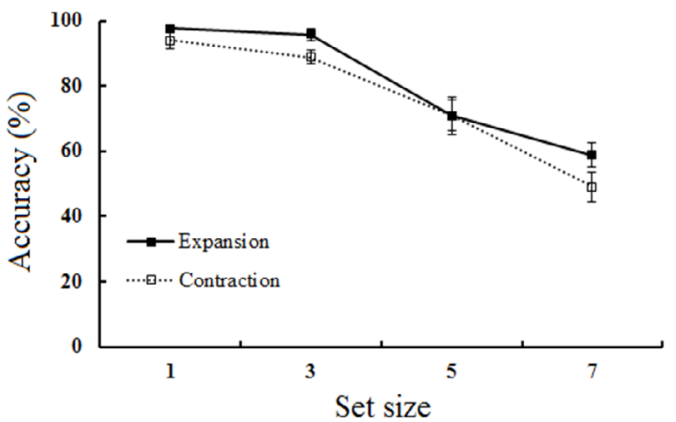

(b)

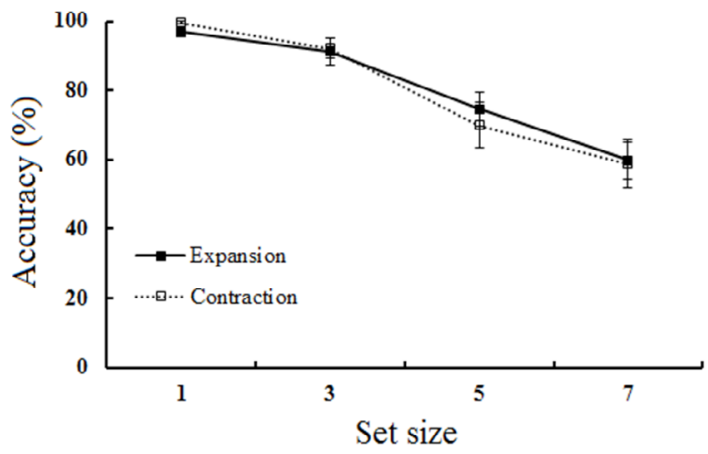

(c)

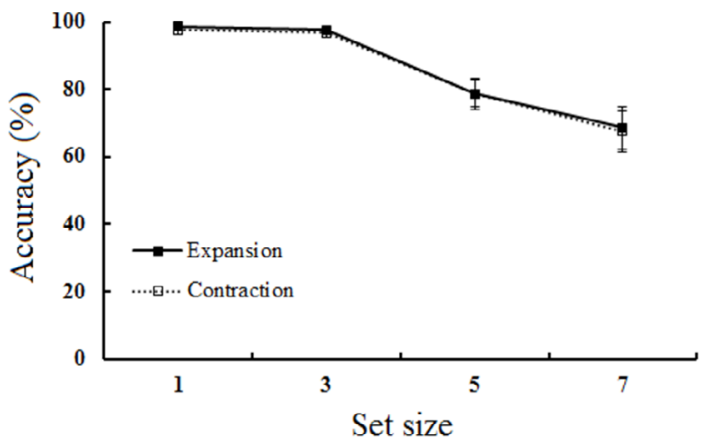

|FIGURE 5.

Mean accuracy of Experiment 2A $(n=9)$ in the congruous $(a)$, incongruous (b), and static conditions (c). Error bars denote the standard error of means. The solid line represents the accuracy for expanding motion and the dotted line represents the accuracy for contracting motion. when the cueing effect cannot be observed, because the decision load does not relate to the modulation.

In Experiment 2B, we considered the prediction with the expansion benefit as follows. If the modulation of expanding and contracting motions is caused in the retrieval (decision) stage, the expansion benefit may not be observed (or weaken the expansion benefit) because the cueing stimulus reduces the amount of decision load and enhances memory performance for both expanding and contracting motions. Thus, memory performance for expanding and contracting motions may be similar. On the other hand, if the modulation of expanding and contracting motions is not caused in the retrieval stage, then the expansion benefit may be observed in a manner similar to the previous experiments, because the cueing stimulus (i.e., decision load) cannot affect memory performance.

\section{Experiment 2B}

\section{METHOD}

Seven people (two men and five women) took part in Experiment 2B (three new participants), all of whom were naive to the purpose of the study. The same design as in Experiment 1A was used, with the exception that we added a cue condition (no cue or cue). Each trial in the no cue condition was identical to those in Experiment 1A. In contrast, for the cue trials, a white frame $\left(64.0 \mathrm{~cd} / \mathrm{m}^{2}\right)$ appeared in place of a single test item for a $200 \mathrm{~ms}$ period prior to the presentation of the test display (see Figure 6, Panel A). In addition, we used only Set sizes of 5 and 7 objects in this experiment.

The three factors of motion (expansion or contraction), set size ( 5 or 7 ), and cueing (no cue or cue) were examined. Therefore, each participant completed 320 trials $(2$ motions $\times 2$ set sizes $\times 2$ cue settings $\times 2$ conditions [same or different] $\times 20$ trials), with the cue condition randomized within each participant.

\section{RESULTS AND DISCUSSION}

Figure 6, Panel B shows the accuracy across all participants in Experiment 2B. We conducted a three-way ANOVA on the factors of motion, set size, and cueing. The results showed a significant main effect of cueing, $F(1,6)=22.53, p=.003, \eta_{p}{ }^{2}=.79$, and set size, $F(1,6)=31.94, p=.001, \eta_{\mathrm{p}}^{2}=.84$, and a significant interaction between motion, set size, and cueing, $F(1,6)=7.03, p=.04$, $\eta_{\mathrm{p}}{ }^{2}=.54$. With regard to a simple main effect of cue, the accuracy for cue condition was higher than that for the no cue condition in the expanding motion at Set sizes 5 and $7, F(1,24)=5.22, p=.03$, $\eta_{\mathrm{p}}{ }^{2}=.18$ for Set size 5 and $F(1,24)=6.71, p=.02, \eta_{\mathrm{p}}{ }^{2}=.22$ for Set size 7, as well as for the contracting motion at Set size $7, F(1,24)=$ 33.52, $p=.001, \eta_{\mathrm{p}}{ }^{2}=.58$. A simple main effect of motion was observed in the no cue condition at Set size 7, $F(1,24)=4.74, p=.04$, $\eta_{\mathrm{p}}{ }^{2}=.16$, confirming that the accuracy for expanding motion was higher than that for contracting motion in this condition. Further, a simple main effect of set size was observed for expanding motion in the no cue and cue conditions, $F(1,24)=7.53, p=.01, \eta_{\mathrm{p}}{ }^{2}=.24$ for the no cue condition and $F(1,24)=6.03, p=.02, \eta_{\mathrm{p}}{ }^{2}=.20$ for 
the cue condition, as well as for contracting motion in the no cue condition, $F(1,24)=30.14, p=.001, \eta_{p}{ }^{2}=.56$, confirming that in these conditions, the accuracy for Set size 5 was higher than that for Set size 7 . However, we could not observe a main effect of motion, $F(1,6)=0.17, p=.69, \eta_{\mathrm{p}}{ }^{2}=.03$, an interaction between motion and cueing, $F(1,6)=1.91, p=.22, \eta_{\mathrm{p}}{ }^{2}=.24$, between set size and cueing, $F(1,6)=5.03, p=.07, \eta_{\mathrm{p}}{ }^{2}=.24$, or between motion and set size, $F(1,6)=0.47, p=.52, \eta_{\mathrm{p}}{ }^{2}=.46$.

We calculated the necessary sample size using an effect size (significant interaction among motion, set size, and cueing: $\left.\eta_{\mathrm{p}}{ }^{2}=.54\right)$ with a power level $(1-\beta)$ of 0.8 . We showed that a total sample size of 6 was necessary for obtaining results with an $\alpha$ of 0.05. Thus, our sample size $(n=7)$ is be sufficient for examining the results of Experiment 2B.

Experiment 2B showed that a cueing stimulus enhanced memory performance for both expanding and contracting motion, indicating that the expansion benefit disappeared in the cue condition. Thus, Experiment 2 confirmed that the expansion benefit might occur at the retrieval stage of memory processes. We propose that the contents of the test display would be a critical factor in modulating the expansion benefit.

\section{EXPERIMENT 3: \\ THE EXPANSION BENEFIT IN THE STOR- AGE STAGE OF VISUAL SHORT-TERM MEMORY}

In Experiment 3, we further examined the modulatory effect of expanding and contracting motion in the storage stage of VSTM. To explore a critical factor for the modulatory effect in the storage stage, we manipulated the interstimulus interval (ISI, a blank display in the change-detection task) between the memory and test displays. We predicted that the expansion benefit would be enhanced with increasing ISI because the memory representation for the contracting motion would become weak and attenuated. To examine these possibilities, we manipulated the ISI using a blank display of 400, 800, and $1200 \mathrm{~ms}$ in duration, in contrast to the blank display duration for Experiments 1 and 2, which was fixed at $400 \mathrm{~ms}$.

\section{Method}

Fourteen people (five men and nine women) took part in Experiment 3 (five new participants), all of whom were naive to the purpose of the study. The same design as in Experiment 1A was used, with the exception that we manipulated the ISI (either 400, 800, or $1200 \mathrm{~ms}$ ). For this experiment, we used set sizes of 3,5 , and 7 objects.

(a)

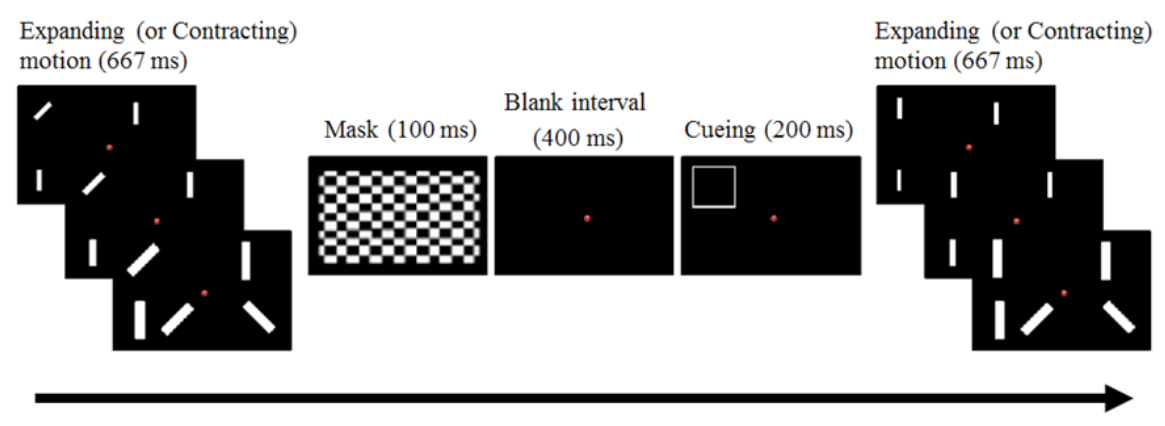

(b)

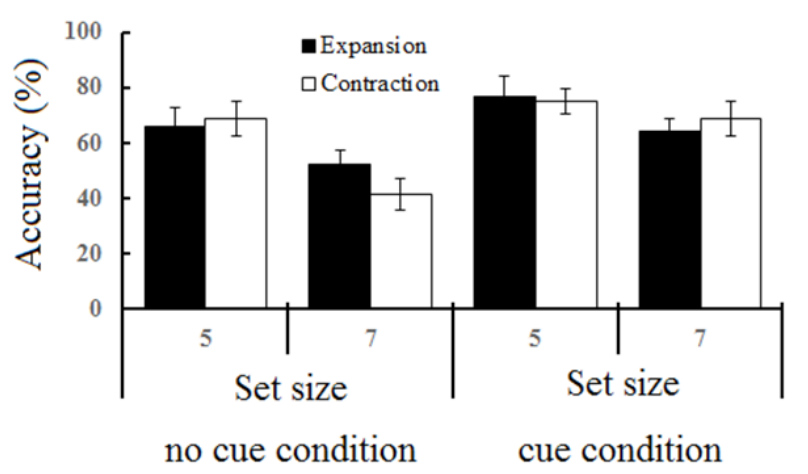

|FIGURE 6.

Panel A: Cueing stimuli used in Experiment 2B. Panel B: Mean accuracy of Experiment 2B for an object set size of $7(n=7)$. Error bars denote the standard error of the means. The black bar represents the accuracy for expanding motion and the white bar represents the accuracy for contracting motion. 
(a)

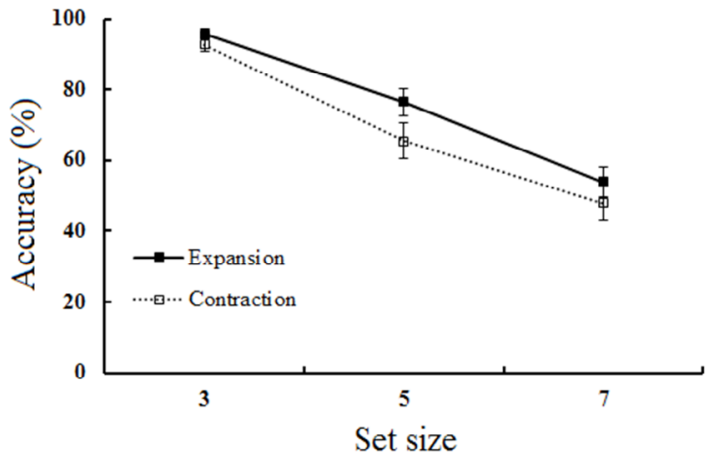

(b)

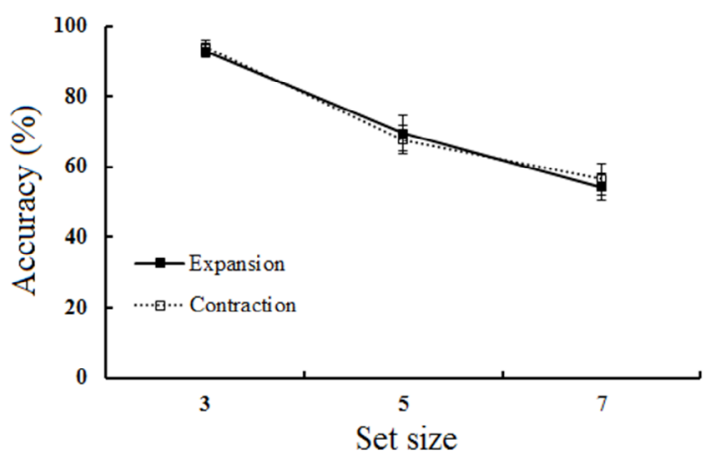

(c)

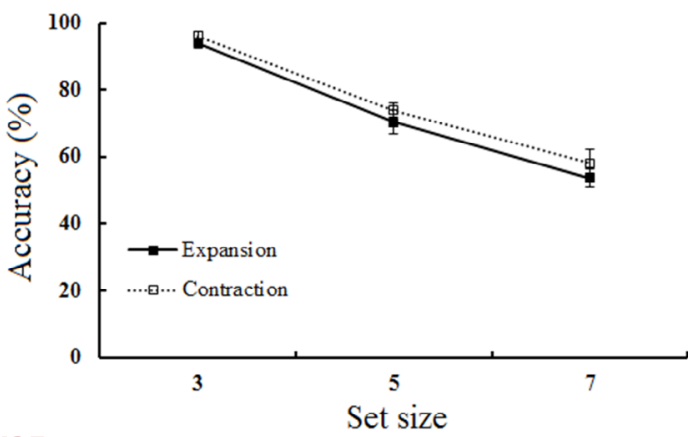

FIGURE 7.

Mean accuracy of Experiment $3(n=14)$ for an ISI of 400 ms (Panel A), 800 ms (Panel B), and 1200 ms (Panel C). Error bars denote the standard error of the means. The solid line represents the accuracy for expanding motion and the dotted line represents the accuracy for contracting motion.

The three factors of motion (expansion or contraction), set size $(3,5$, or 7$)$, and ISI $(400,800$, or $1200 \mathrm{~ms})$ were examined. Therefore, each participant completed 720 trials ( 2 motions $\times 3$ set sizes $\times 3$ ISIs $\times 2$ conditions [same or different] $\times 20$ trials) with the ISI presentation time randomized within each participant.

\section{Results and Discussion}

We conducted a three-way ANOVA on the factors of motion, set size, and ISI (see Figure 7). We observed a significant main effect of set size, $F(2,26)=135.90, p=.001, \eta_{\mathrm{p}}{ }^{2}=.91$. Our post-hoc analysis showed significant differences in all set sizes (Set sizes 3 vs. $5: t[26]=9.67$, $p=.001$; Set sizes 3 vs. $7: t[26]=16.40, p=.001$; Set sizes 5 vs. 7 : $t[26]=6.73, p=.001)$. Moreover, we observed a significant interaction between motion and ISI, $F(2,26)=16.95, p=.001, \eta_{\mathrm{p}}{ }^{2}=.57$. Regarding the interaction, a simple main effect of ISI was observed for contracting motion, $F(2,52)=4.12, p=.004, \eta_{\mathrm{p}}{ }^{2}=.14$. The post-hoc analysis showed the accuracy for the contracting motion at the ISI of $400 \mathrm{~ms}$ was lower than that for the ISI of $1200 \mathrm{~ms}, t(52)=3.50, p=.001$. Also, a simple main effect of motion was observed at ISIs of 400 and 1200 ms, $F(1,39)=23.25, p=.001, \eta_{\mathrm{p}}{ }^{2}=0.25$ for the ISI of $400 \mathrm{~ms}$ and $F(1,39)=6.06, p=.018, \eta_{p}{ }^{2}=.21$ for the ISI of $1200 \mathrm{~ms}$, revealing that the accuracy was smaller for contracting motion than for expanding motion at the ISI of $400 \mathrm{~ms}$ and that this tendency was reversed at the ISI of $1200 \mathrm{~ms}$. However, we could not observe main effects of motion, $F(1,13)=0.97, p=.034, \eta_{\mathrm{p}}^{2}=.07$ and ISI, $F(2,26)=0.86, p=0.43$, $\eta_{\mathrm{p}}{ }^{2}=.06$, interactions between motion and set size, $F(2,26)=0.59$, $p=.56, \eta_{\mathrm{p}}^{2}=.04$, between set size and ISI, $F(4,52)=1.66, p=.17$, $\eta_{\mathrm{p}}{ }^{2}=.11$, or among motion, set size, and ISI, $F(4,52)=0.81, p=.53, \eta_{\mathrm{p}}{ }^{2}=.06$.

To confirm whether our sample size was sufficient for examining the results of Experiment 3, we calculated the necessary sample size using an effect size (significant interaction between motion and ISI: $\left.\eta_{\mathrm{p}}{ }^{2}=.57\right)$ with a power level $(1-\beta)$ of 0.8 . We found that a total sample size of 6 was necessary to obtain the results with an $\alpha$ of 0.05 . We assumed that our sample size $(n=14)$ would be sufficient for examining the results of Experiment 3.

Experiment 3 replicated the expansion benefit at the ISI of $400 \mathrm{~ms}$. The memory performance for contracting motion increased for the longer ISI $(1200 \mathrm{~ms})$, although the memory performance for expanding motion did not vary between ISIs of 400 and $1200 \mathrm{~ms}$. As a result, the expansion benefit disappeared at the longer ISI. These findings suggest that the storage stage might affect the occurrence of the expansion benefit; ISI is an important factor for the modulatory effect of expanding and contracting motions in terms of the storage stage. Also, because time may be needed to store the memory representation for contracting motion, there may be differences in the memory performance between expanding and contracting motions at the longer ISI.

\section{EXPERIMENT 4:}

\section{THE EXPANSION BENEFIT IN THE EN- CODING STAGE OF VISUAL SHORT-TERM MEMORY}

We next considered the other possibility that the modulatory effect might be observed in the early encoding stage of VSTM. To examine this possibility, in Experiment 4, we manipulated the presentation time of the memory display in the change-detection task. To sufficiently encode a memory representation of a stimulus, the presentation time may need to be greater than $500 \mathrm{~ms}$ (Alvarez \& Cavanagh, 2004). If the modulatory effect of expanding and contracting motions occurs in the encoding stage of VSTM, and if a shorter presentation time for the memory display is used, memory performance may decrease more for contracting motion than for expanding motion because memory representation for contracting motion may not be sufficiently encoded. Therefore, we manipulated the presentation time for the memory display to be less than $500 \mathrm{~ms}$ (417 ms), compared to the presentation time of $667 \mathrm{~ms}$, as used in Experiments 1 through 3. 
(a)

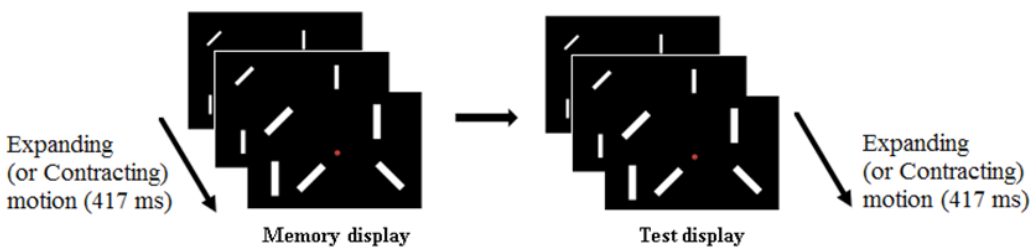

(b)

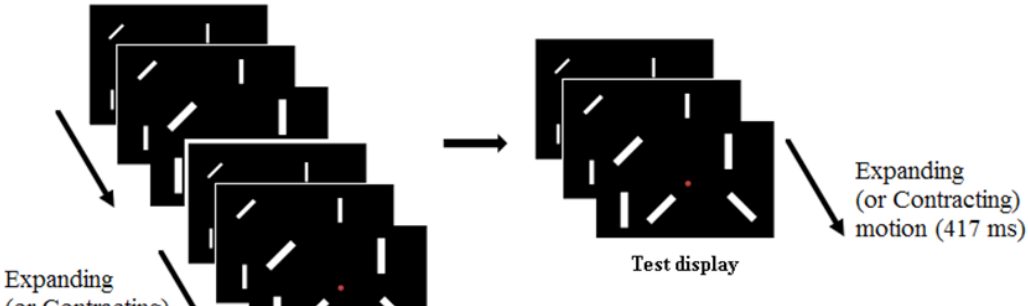

FIGURE 8.
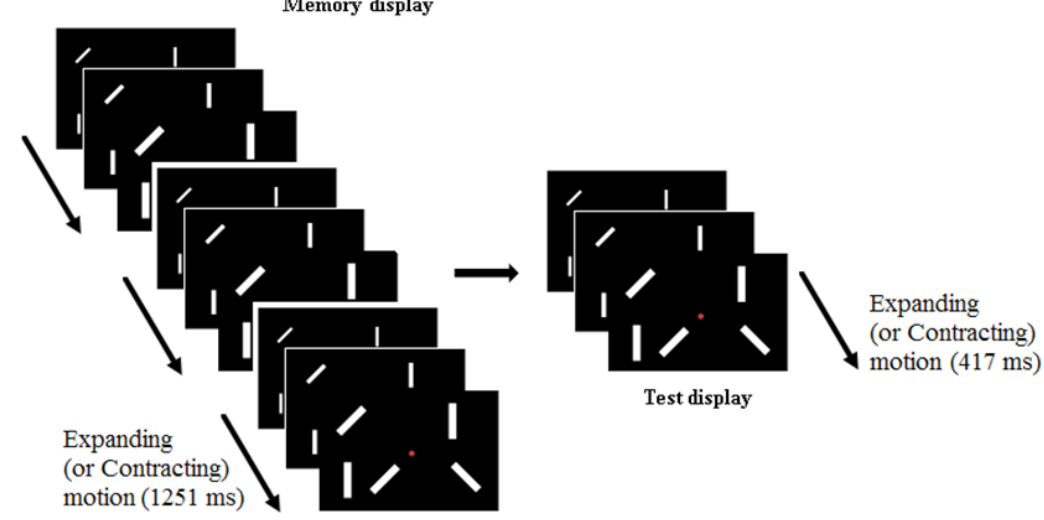

Test display

Samples of stimuli used to determine the effect of presentation times of the memory display in Experiment 4. Presentation times of 417 ms (Panel A), 834 ms (Panel B), and 1251 ms (Panel C). In each trial with presentation times of 834 and 1251 ms, the presentation time of 417 ms with expanding (or contracting) motion was repeated twice or three times, respectively.

\section{Method}

Eleven people (two men and nine women) took part in Experiment 4 (five new participants), all of whom were naive to the purpose of the study. The same design as in Experiment 1A was used, with the exception that we manipulated the presentation time for the memory display $(417,834$, or $1251 \mathrm{~ms})$. In each trial with a presentation time of $417 \mathrm{~ms}$, line segments with expanding (or contracting) motion were presented for $417 \mathrm{~ms}$ per trial whereas in each trial with presentation times of 834 and $1251 \mathrm{~ms}$, the presentation time of $417 \mathrm{~ms}$ with expanding (or contracting) motion was repeated twice and thrice, respectively (see Figure 8). For this experiment, we used set sizes of 3, 5, and 7 objects. The three factors of motion (expansion or contraction), set size $(3,5$, or 7$)$, and presentation time $(417,834$, or $1251 \mathrm{~ms})$ were examined. Therefore, each participant completed 720 trials ( 2 motions $\times 3$ set sizes $\times 3$ presentation times $\times 2$ conditions [same or different] $\times 20$ trials), with the presentation time randomized within each participant.

\section{Results and Discussion}

We conducted a three-way ANOVA on the factors of motion, set size, and presentation time (see Figure 9). We found significant main effects of set size, $F(2,20)=157.72, p=.001, \eta_{\mathrm{p}}{ }^{2}=.94$. The post-hoc analysis showed significant differences in all set sizes (see Supplementary Table 4). Moreover, a significant interaction between motion and set size was observed, $F(2,20)=3.97, p=.04, \eta_{\mathrm{p}}{ }^{2}=.28$. A simple main effect of motion was observed in Set size $7, F(1,30)=7.38, p=.01, \eta_{\mathrm{p}}{ }^{2}=.20$, confirming that the accuracy for expanding motion was higher than that for contracting motion in Set size 7. Also, a simple main effect of set size was observed in both expanding and contracting motions, $F(2,40)=85.87, p=.01, \eta_{\mathrm{p}}{ }^{2}=.81$ for expanding motion and $F(2,40)=131.88, p=.01, \eta_{\mathrm{p}}{ }^{2}=.87$ for contracting motion. The posthoc analysis showed significant differences in all set sizes for expansion and contraction (see Supplementary Table 4). However, we could not observe main effects of presentation time, $F(2,20)=2.05, p=.15$, $\eta_{\mathrm{p}}{ }^{2}=.17$ and motion, $F(1,10)=2.81, p=.12, \eta_{\mathrm{p}}{ }^{2}=.22$, interactions between motion and presentation time, $F(2,20)=1.79, p=.19, \eta_{\mathrm{p}}{ }^{2}=.16$, between set size and presentation time, $F(4,40)=1.87, p=.14, \eta_{\mathrm{p}}{ }^{2}=.16$, or among motion, set size, and presentation time, $F(4,40)=1.90, p=.13$, $\eta_{\mathrm{p}}^{2}=.16$.

To confirm the necessary sample size, we calculated it using an effect size (significant interaction between motion and set size: $\eta_{\mathrm{p}}{ }^{2}=.28$ ) with a power level $(1-\beta)$ of 0.8 . We found that a total sample size of 6 was necessary for obtaining results with an $\alpha$ of 0.05 . Thus, our sample size $(n=11)$ was sufficient for Experiment 4. 
Experiment 4 showed no effect of the presentation time on memory performance between expanding and contracting motion (i.e., in all presentation times, we observed the expansion benefit, which was not be affected by any presentation time). This implies that the encoding stage of VSTM may not affect the expansion benefit.

\section{GENERAL DISCUSSION}

The goal of the current study was to reveal the modulatory effect of expanding and contracting motions on VSTM by using a change-detection task. Experiment 1A showed that memory performance was higher when the objects contained expanding motion than when they contained contracting motion (the expansion benefit), which were observed only when we compared memory performance between expanding and contracting motion, that is, memory performance for objects that contained motion (both expansion and contraction) were lower compared with objects

(a)

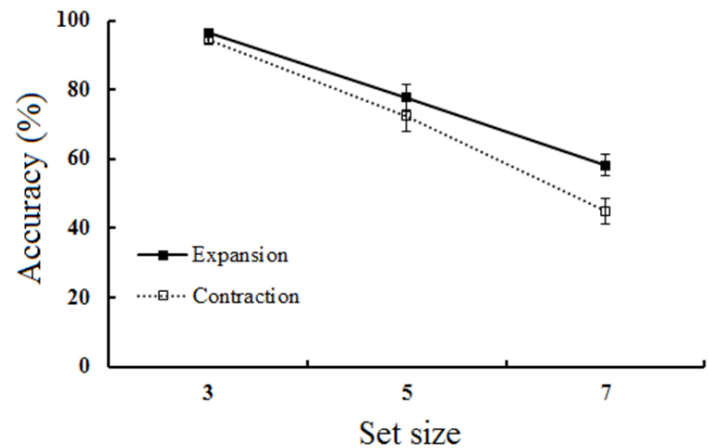

(b)

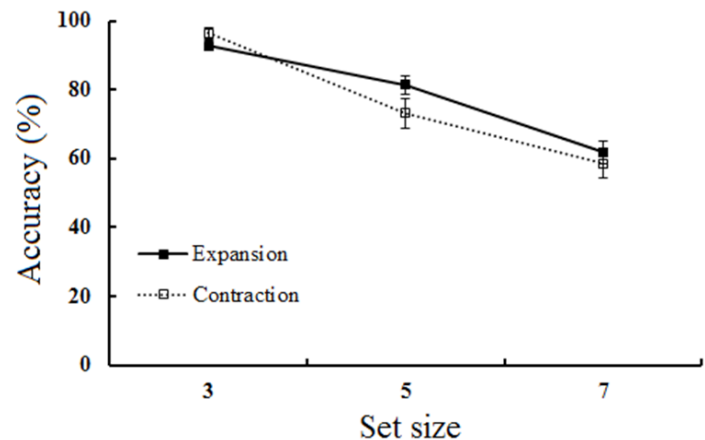

(c)

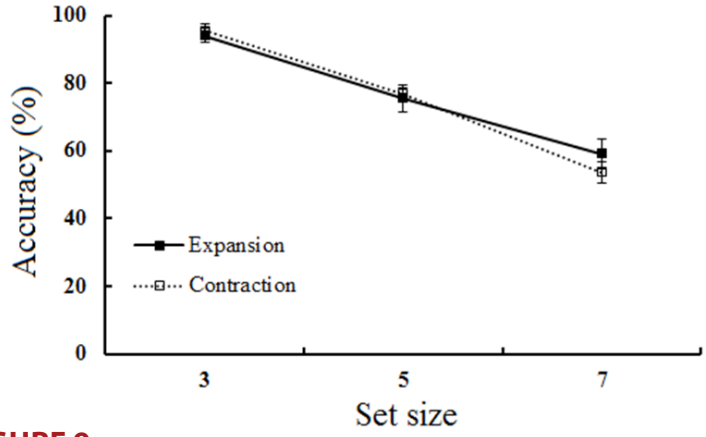

FIGURE 9.

Mean accuracy of Experiment $4(n=11)$ for the presentation times of 417 ms (anel A), 834 ms (Panel B), and 1251 ms (Panel C). Error bars denote the standard error of the means. The solid line represents the accuracy for expanding motion and the dotted line represents the accuracy for contracting motion. that were static (without motion). Experiments 2 to 4 examined the expansion benefit in terms of specific VSTM processing stages (encoding, storage, and retrieval) to explore which factors were critical at each stage. Experiment 2 focused on the retrieval stage: Experiment 2A manipulated motion congruency between the memory and test displays and replicated the expansion benefit only when the test display contained the same type of (contracting) motion as the memory display. Experiment 2B showed a cueing effect in the test display whereby cueing a stimulus enhanced memory performance for both expanding and contracting motions, suggesting that the expansion benefit disappeared in the cue condition. These findings (Experiments 2A and 2B) suggested that the contents of the test display are a modulatory factor for the expansion benefit in the retrieval stage. Experiment 3 focused on the storage stage: We manipulated the ISI between the memory and test displays using a blank display with different durations of presentation $(400,800,1200 \mathrm{~ms})$, and replicated the expansion benefit only with an ISI of $400 \mathrm{~ms}$. The memory performance for objects with expanding motion decreased with an increased ISI, leading to a higher memory performance for objects with contracting motion, rather than expanding motion, at an ISI of $1200 \mathrm{~ms}$. These findings suggested that the ISI between the memory and test displays was a modulatory factor for the expansion benefit in the storage stage. Experiment 4 investigated the encoding stage: We manipulated the presentation time of the memory display (durations of 417, 834, and $1251 \mathrm{~ms}$ ) and replicated the expansion benefit in all presentation times. This means that any presentation times could not modulate the expansion benefit.

\section{Observed Expansion Benefit in the Visual Short-Term Memory}

The present study observed a higher memory performance for objects with expanding motion than those with contracting motion (the expansion benefit). Experiment $1 \mathrm{~A}$ also showed that the memory performance for both expanding and contracting motion was lower than that for static line segments (without motion), suggesting that the expansion benefit was observed only when we compared memory performances between expanding and contracting motions. Motion information carries the additional cost of spatiotemporal updating (Saiki \& Miyatsuji, 2009). As such, expanding and contracting motions might incur additional costs to storage because of motion information, leading to a reduced memory performance for objects with expanding and contracting motions than for static line segments. In this experimental setting, which compared memory performance between expanding and contracting motions, we observed the expansion benefit.

\section{The Expansion Benefit is Modulated Depending on Each Stage of Visual Short-Term Memory Processing}

To examine the expansion benefit in more detail, further experiments focused on specific VSTM processing stages (encoding, storage, and retrieval). The VSTM processing is generally considered to occur as follows: A stimulus is encoded as an available representation in VSTM (i.e., the encoding stage); this representation is temporally activated and updated in 
VSTM (i.e., the storage stage). Observers compare this memory representation with newly presented stimuli, leading to an identification as either the same or different (i.e., the retrieval stage). We explored modulatory factors for the expansion benefit for each stage of VSTM processing.

Regarding the retrieval stage (Experiments $2 \mathrm{~A}$ and $2 \mathrm{~B}$ ), we manipulated the contents of the test display in the change-detection task. Experiment 2A involved manipulating the motion congruency between the memory and test displays (congruous, incongruous, and static conditions). Results showed that the expansion benefit was observed only in the congruous condition. Experiment 2A further showed that the memory performance for objects with contracting motion under the congruous condition was lower than the static line conditions, although there were no significant differences in the memory performance for expanding motion between congruous, incongruous, and static conditions. Although precisely what was stored in VSTM was common across the motion congruency conditions (i.e., the memory display was unchanged), the memory performance for contracting motion varied depending on the condition of motion congruency. Thus, the dependency of memory performance on the contents of the test display in this experiment was evidence of an effect at the retrieval stage of memory processing (Wheeler \& Treisman, 2002). The expansion benefit might be observed because of lower memory performance for contracting motion. Based on this suggestion, our results for expanding and contracting motions might be interpreted as follows: Since the motion information carries the additional cost of spatiotemporal updating (Saiki \& Miyatsuji, 2009), the congruent and incongruent conditions might show a heavier cognitive load than the static line conditions. Moreover, contracting motion might have a heavier cognitive load compared with expanding motion. From these accounts, although memory representation for contracting motion might be relatively weak, the test display that included expanding motion (the incongruent condition) ameliorated this weakness. Thus, we observed the expansion benefit only in the congruent condition, not in the incongruent and static line conditions. Experiment $2 \mathrm{~B}$ added a cueing stimulus to the test display. If the contents of the test display modulated the expansion benefit found in Experiment 2A, a cueing stimulus might similarly reduce this benefit since cueing stimuli generally reduce the amount of decision load and enhance memory performance (Wheeler \& Treisman, 2002; Yang et al., 2015). As predicted, there was no expansion benefit in Experiment 2B with the cueing condition. We can conclude that the expansion benefit might be affected by the display contents used at the retrieval stage.

Regarding the storage stage (Experiment 3), we manipulated the ISI using a blank display in the change-detection task. The expansion benefit was replicated only with an ISI of $400 \mathrm{~ms}$ (as used in Experiments 1 and 2): the effect was not observed with an ISI of more than $800 \mathrm{~ms}$. With an ISI of $800 \mathrm{~ms}$, there was no difference in memory performance between objects with expanding and contracting motions, whereas with an ISI of $1200 \mathrm{~ms}$, memory performance was lower for expanding motion than for contracting motion. The memory performance for the expanding motion did not vary due to the ISIs of 400, 800, and $1200 \mathrm{~ms}$. In contrast, the memory performance for the contracting motion with an ISI of $1200 \mathrm{~ms}$ was higher than that with $400 \mathrm{~ms}$. We suggest that memory representations for contracting motion might become stronger with longer ISIs due to the additional time needed to store memory representations for contracting motion, leading to the expansion benefit only at a short ISI (400 ms).

Regarding the encoding stage (Experiment 4), we manipulated the presentation time of the memory display. We found the expansion benefit in all presentation times, suggesting that no presentation time could modulate the expansion benefit, and thus, the expansion benefit may not be affected in the encoding stage of VSTM. However, one might argue that our experimental settings could not fully manipulate the presentation time, leading to a null effect of presentation time on the expansion benefit. In our present experimental settings, an expanding (or contracting) motion was repeatedly presented to manipulate the presentation time (specifically, 2 and 3 times of motion frames were presented for 834 and $1251 \mathrm{~ms}$, respectively). This means that each expanding (or contracting) motion included its opposite motion component when the motions were repeatedly presented, which might induce an apparent motion (see also Takeuchi, 1997). Considering other assumptions, we assumed that because orientation was a simple feature which might not need to higher cognitive load, the presentation time would not affect to the expansion benefit. To examine this possibility in the future, other experimental stimuli that include higher cognitive loads or other complex features should be utilized. Since the experimental settings of Experiment 4 might not have manipulated temporal properties, from these views we must carefully conclude a null effect of presentation time on the expansion benefit.

We then interpreted our results based on each VSTM processing as follows. In the encoding stage, the expansion benefit was observed regardless of the manipulation of presentation time. Since the visual system preferentially detects expanding rather than contracting motion (Takeuchi, 1997), memory representations for expanding motion may be more efficiently formed. In the storage stage, although the expansion benefit was observed in the shorter ISI (400 ms), this effect disappeared in the longer ISIs (800 and $1200 \mathrm{~ms}$ ) because memory representation for contracting motion was sufficiently formed during these time windows. Therefore, the memory representation for expanding motion might have been formed quickly in the encoding stage and continuously stored during the storage stage, whereas the memory representation for contracting motion might have been formed slowly during the encoding stage, becoming stronger with longer ISIs due to the additional time needed in the storage stage to store its memory representations. In the retrieval stage, the expansion benefit in which the test display of change-detection task resulted in lower cognitive load manipulation (e.g., the motion congruency between displays and a cueing stimulus) could be observed. Thus, the expansion benefit was manipulated by contents (cognitive load) of the test display.

Previous studies have reported the modulation of expanding and contracting motions on visual cognition in terms of detection or visual search (Takeuchi, 1997) and capturing visual attention (Franconeri \& Simons, 2003; Kawahara et al., 2012), which means that the effects were observed in the comparatively lower level of visual cognition. The results of the present study demonstrated the modulation of expanding and contracting motions on VSTM, which can be considered a comparatively higher level of visual cognition. In light of our new findings, we propose that the modulation of expanding and contracting motion can be observed in 
the higher level of visual cognition in addition to the lower level of visual cognition.

\section{The Expansion Benefit From a Psychological Point of View}

Here we consider the results from a physiological point of view. Middle temporal area (MT) cells are sensitive to the direction of translation while middle superior temporal area (MST) cells are sensitive to more complex motions, such as expanding and contracting motions (Graziano, Andersen, \& Snowden, 1994; Tanaka \& Saito, 1989; Vaina, 1998). Tanaka and Saito showed that the expanding and contracting motion-sensitive cells are concentrated within MST cell populations and that in macaque monkeys, the number of cells that selectivity respond to expanding motion is greater than the number that respond selectivity to contracting motion. Graziano et al. reported similar findings. Based on these studies, we suggest that the present results might be explained by a numerical difference between expanding and contracting motion cells in the MST. The MT/MST cells are also activated by the temporary storage of expanding and contracting objects, as well as the processing of these objects (Bisley \& Pasternak, 2000; Bisley, Zaksas, Droll, \& Pasternak, 2004). Bisley et al. showed that the MT cells of monkeys display sustained activations during the delay periods of a short-term memory task with a requirement to store moving stimuli. The present results might provide possible evidence that the MT/MST cells, corresponding to the human motion complex $(\mathrm{hMT}+)$, affect not only expanding and contracting motion processing but also the temporary storage of this information.

\section{An Ecological Explanation of the Expansion Benefit}

It is plausible to assume an ecological explanation for our findings, as previous studies have suggested (e.g., Shirai et al., 2004, 2008, 2009; Takeuchi, 1997). Takeuchi reported that to avoid approaching objects that can represent dangerous situations, such as collisions, the visual system is specialized to detect expanding motion. In the context of VSTM research, Jackson et al. (2009) showed that the memory performance for high threat objects (e.g., angry faces) was enhanced compared with low threat objects (e.g., happy or neutral faces), suggesting that the visual system is specialized to make rapid, effective decisions of whether to approach or avoid threatening objects represented in VSTM. Previous studies (Jackson et al., 2009; Öhman et al., 2001) have pointed out that the visual system can more quickly allocate visual attention, often thought of as the gateway to VSTM, to high threat objects compared with low threat objects. When visual attention is directed to behaviorally relevant stimuli (i.e., high threat objects), the stimuli are processed efficiently while behaviorally irrelevant stimuli are filtered out (biased competition model, Desimone, 1998). Thus, visual attention allocated to high threat objects can enhance the encoding and temporary storage of their representations in VSTM, potentially inhibiting the processing of low threat objects. Based on these ecological explanations (Takeuchi, 1997; Jackson et al., 2009), we can assume that the visual system rapidly deals with expanding objects that potentially signal dangerous situations such as collisions, and that they are encoded more rapidly and efficiently than are contracting objects. Moreover, to decide whether to approach or avoid these situations, the visual system must store the correct representations of objects in VSTM. Thus, the memory performance for expanding objects might be larger than that for contracting objects. However, we should also note the bias for contracting motion. Several previous studies (Edwards \& Badcock, 1993; Edwards \& Ibbotson, 2007; Shirai et al., 2004, 2006, 2009) indicated that the visual system may show a higher sensitivity to contracting motion than expanding motion. For example, Shirai et al. (2009) measured sensitivity for expanding and contracting motions using a visual evoked potential (VEP) method with a first harmonic as an indicator in 3-months-old, 4-months-old, and adult participants. They compared the amplitude of the first harmonic between expanding and contracting motions, revealing that the amplitude of the contracting motion was higher than that of the expanding motion in 4-months-old and adult participants. In 4-months-old and adult participants, sensitivity for contracting motion was higher than that for expanding motion. Considering the results of Experiment 3, we also found a contraction benefit compared with expanding motion in the longer ISI (1200 ms). We assumed the possibility that the bias for contracting motion may also be observed in VSTM processes, manipulating the time properties in future research.

\section{CONCLUSION}

We demonstrated the expansion benefit compared with contraction in VSTM. Specifically, we assumed that the expansion benefit might be modulated depending on the stages of VSTM processing. Future research needs to determine whether various ecological and affective properties induced by visual motion can further modulate VSTM. These findings may specify new aspects of VSTM, such as the temporary storage system process for object features, as well ecological and affective meanings.

\section{REFERENCES}

Alvarez, G. A. \& Cavanagh, P. (2004). The capacity of visual shortterm memory is set both by total information load and by number of objects. Psychological Science, 15, 106-111. doi: 10.1111/j.0963-7214.2004.01502006.x x

Alvarez, G. A. \& Cavanagh, P. (2008). Visual short-term memory operates more efficiently on boundary features than on surface features. Perception \& Psychophysics, 70, 346-364. doi: 10.3758/PP.70.2.346 Wلس

Bisley, J. W. \& Pasternak, T. (2000). The multiple roles of visual cortical areas MT/MST in remembering the direction of visual motion. Cerebral Cortex, 10, 1053-1065. doi: 10.1093/ cercor/10.11.1053

Bisley, J. W., Zaksas, D., Droll, J. A., \& Pasternak, T. (2004). Activity of neurons in cortical area MT during a memory for motion task. Journal of Neurophysiology, 91, 286-300. doi: 10.1152/ jn.00870.2003 الس السلسلس

Cohen, J. (1992). A power primer. Psychological Bulletin, 112, $155-159$.

Desimone, R. (1998). Visual attention mediated by biased competition in extrastriate visual cortex. Philosophical Transactions 
of the Royal Society B: Biological Sciences, 353, 1245-1255. doi: 10.1098/rstb.1998.0280 |سلس

Edwards, M. \& Badcock, D. R. (1993). Asymmetries in the sensitivity to motion in depth: A centripetal bias. Perception, 22, 1013-1023. doi: 10.1068/p221013 المالسلس

Edwards, M. \& Ibbotson, M. R. (2007). Relative sensitivities to large-field optic-flow patterns varying in direction and speed. Perception, 36, 113-124. doi: 10.1068/p5626 المالسلس

Fougnie, D., Suchow, J. W., \& Alvarez, G. A. (2012). Variability in the quality of visual working memory. Nature Communications, 3,

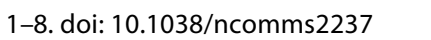

Franconeri, S. L., Alvarez, G. A., \& Cavanagh, P. (2013). Flexible cognitive resources: competitive content maps for attention and memory. Trends in Cognitive Sciences, 17, 134-141. doi: 10.1016/j.tics.2013.01.010 سلس

Franconeri, S. L. \& Simons, D. J. (2003). Moving and looming stimuli capture attention. Perception \& Psychophysics, 65, 999-1010.

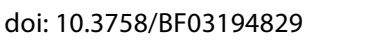

Graziano, M. S. A., Anderson, R. A., \& Snowden, R. J. (1994). Tuning of MST neurons to spiral motions. Journal of Neuroscience, 14,

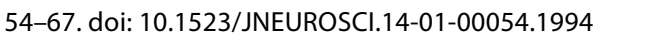

Jackson, M. C. \& Raymond, J. E. (2008). Familiarity enhances visual working memory for faces. Journal of Experimental Psychology: Human Perception and Performance, 34, 556-568. doi: 10.1037/0096-1523.34.3.556 سلس

Jackson, M. C., Wolf, C., Johnston, S. J., Raymond, J. E., \& Linden, D. E. J. (2008). Neural correlates of enhanced visual short-term memory for angry faces: An fMRI study. PloS One, 3, 1-10. doi:

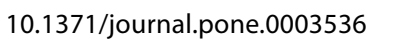

Jackson, M. C., Wu, C-Y., Linden, D. E. J., \& Raymond, J. E. (2009). Enhanced visual short-term memory for angry faces. Journal of Experimental Psychology: Human Perception and Performance, 35, 363-374. doi: 10.1037/a0013895 المبلسلسل

Jiang, Y. V., Olson, I. R., \& Chun, M. M. (2000). Organization of visual short-term memory. Journal of Experimental Psychology: Learning, Memory, and Cognition, 3, 683-702. doi: 10.1037/0278-7393.26.3.683

Jiang, Y. V., Shim, W. M., \& Makovski, T. (2008). Visual working memory for line orientations and face identities. Perception \& Psychophysics, 70, 1581-1591. doi: 10.3758/PP.70.8.1581 المالسلسل

Kawahara, J., Yanase, K., \& Kitazaki, M. (2012). Attentional capture by the onset and offset of motion signals outside the spatial focus of attention. Journal of Vision, 12, 1-13. doi: 10.1167/12.12.10 سلس

Luck, S. J. \& Vogel, E. K. (1997). The capacity of visual working memory for features and conjunctions. Nature, 390, 279-281. doi: 10.1038/36846 السلسلس

Mather, M., Mitchell, K. J., Raye, C. L., Novak, D. L., Greene, E. J., \& Johnson, M. K. (2006). Emotional arousal can impair feature binding in working memory. Journal of Cognitive Neuroscience,

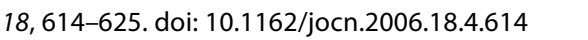

McKeefry, D. J., Burton, M. P., \& Vakrou, C. (2007). Speed selectivity in visual short term memory for motion. Vision Research, 47, 2418-2425. doi: 10.1016/j.visres.2007.05.011 السلسلس

Öhman, A., Flykt, A., \& Esteves, F. (2001). Emotion drives attention: Detecting the snake in the grass. Journal of Experimental Psychology: General, 130, 466-478. doi: 10.1037/00963445.130.3.466 الس الس

Pasternak, T. \& Zaksas, D. (2003). Stimulus specificity and temporal dynamics of working memory for visual motion. Journal of Neurophysiology, 90, 2757-2762. doi: 10.1152/jn.00422.2003 السلسلسلة Phillips, W. A. (1974). On the distinction between sensory storage and visual short-term visual memory. Perception \& Psychophysics, 16, 283-290. doi: 10.3758/BF03203943 |سلس

Saiki, J. \& Miyatsuji, H. (2009). Estimated capacity of object files in visual short-term memory is not improved by retrieval cueing. Journal of Vision, 9, 1-15. doi: 10.1167/9.3.16 المالسلسل

Sheth, B. R. \& Pham, T. (2008). How emotional arousal and valence influence access to awareness. Vision Research, 48, 2415-2424. doi: 10.1016/j.visres.2008.07.013 السلسلسل

Shirai, N., Birtles, D., Wattam-Bell, J., Yamaguchi, M. K., Kanazawa, S., Atkinson, J., \& Braddick, O (2009). Asymmetrical cortical processing of radial expansion/contraction in infants and adults. Developmental Science, 12, 946-955. doi: 10.1111/j.1467-

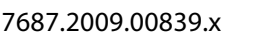

Shirai, N., Kanazawa, S., \& Yamaguchi, M. K. (2004). Asymmetry for the perception of expansion/contraction in infancy. Infant Behavior \& Development, 27, 315-322. doi: 10.1016/j.infbeh.2003.12.004 السلسلس

Shirai, N., Kanazawa, S., \& Yamaguchi, M. K. (2006). Anisotropic motion coherence sensitivities to expansion/contraction motion in early infancy. Infant Behavior \& Development, 29, 204-209. doi: 10.1016/j.infbeh.2005.10.003 المالسلس

Shirai, N., Kanazawa, S., \& Yamaguchi, M. K. (2008). Early development of sensitivity to radial motion at different speeds. Experimental Brain Research, 185, 461-467. doi: 10.1007/ s00221-007-1170-2 سلس

Shirai, N. \& Yamaguchi, M. K. (2004). Asymmetry in the perception of motion-in-depth. Vision Research, 44, 1003-1011. doi: 10.1016/j.visres.2003.07.012 المالس

Takahashi, J., Kawachi, Y., \& Gyoba, J. (2015). Visual short-term memory is modulated by visual preference for spatial configuration between objects. Gestalt Theory, 37, 141-160. السلسلس

Takeuchi, T. (1997). Visual search of expansion and contraction. Vision Research, 37, 2083-2090. doi: 10.1016/S00426989(96)00225-8 سلس

Tanaka, K. \& Saito, H. (1989). Analysis of motion of the visual field by direction, expansion/contraction, and rotation cells clustered in the dorsal part of the medial superior temporal area of the macaque monkey. Journal of Neurophysiology, 62, 626-641. doi: 10.1152/jn.1989.62.3.626 السلسلس

Vaina, L. M. (1998). Complex motion perception and its deficits. Current Opinion in Neurobiology. 8, 494-502. doi: 10.1016/ S0959-4388(98)80037-8 السلسلس 
Wheeler, M. E. \& Treisman, A. M. (2002). Binding in short-term visual memory. Journal of Experimental Psychology: General,

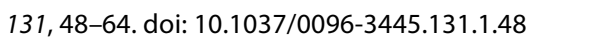

Yang, C-T., Tseng, P., \& Wu, Y-J. (2015). Effect of decision load on whole-display superiority in change detection. Attention, Perception, \& Psychophysics, 77, 749-758. doi: 10.3758/s13414015-0834-4 |لسلس
Zaksas, D., Bisley, J. W., \& Pasternak, T. (2001). Motion information is spatially localized in a visual working-memory task. Journal of Neurophysiology, 86, 912-921. doi: 10.1152/jn.2001.86.2.912

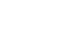

RECEIVED 18.10.2018| ACCEPTED 1.06.2019 


\section{APPENDIX}

\section{SUPPLEMENTARY TABLE 1.}

Experiment 1A, Results of Post Hoc Tests

\begin{tabular}{|c|c|c|}
\hline & $t$ & $p$ \\
\hline \multicolumn{3}{|l|}{ Simple main effect of motion } \\
\hline \multicolumn{3}{|l|}{ Set size 5} \\
\hline expansion vs. contraction & $t(88)=3.98$ & .001 \\
\hline expansion vs. without motion & $t(88)=2.20$ & .001 \\
\hline contraction vs. without motion & $t(88)=6.19$ & .001 \\
\hline \multicolumn{3}{|l|}{ Set size 7} \\
\hline expansion vs. contraction & $t(88)=3.36$ & .001 \\
\hline expansion vs. without motion & $t(88)=4.09$ & .001 \\
\hline contraction vs. without motion & $t(88)=7.44$ & .001 \\
\hline \multicolumn{3}{|l|}{ Simple main effect of Set size } \\
\hline \multicolumn{3}{|l|}{ Expansion } \\
\hline Set sizes 1 vs. 5 & $t(99)=7.11$ & .001 \\
\hline Set sizes 1 vs. 7 & $t(99)=13.40$ & .001 \\
\hline Set sizes 3 vs. 5 & $t(99)=5.08$ & .001 \\
\hline Set sizes 3 vs. 7 & $t(99)=11.37$ & .001 \\
\hline Set sizes 5 vs. 7 & $t(99)=6.29$ & .001 \\
\hline \multicolumn{3}{|l|}{ Contraction } \\
\hline Set sizes 1 vs. 5 & $t(99)=10.05$ & .001 \\
\hline Set sizes 1 vs. 7 & $t(99)=15.74$ & .001 \\
\hline Set sizes 3 vs. 5 & $t(99)=8.63$ & .001 \\
\hline Set sizes 3 vs. 7 & $t(99)=14.31$ & .001 \\
\hline Set sizes 5 vs. 7 & $t(99)=5.69$ & .001 \\
\hline \multicolumn{3}{|l|}{ Without motion } \\
\hline Set sizes 1 vs. 5 & $t(99)=4.77$ & .001 \\
\hline Set sizes 1 vs. 7 & $t(99)=9.24$ & .001 \\
\hline Set sizes 3 vs. 5 & $t(99)=4.47$ & .001 \\
\hline Set sizes 3 vs. 7 & $t(99)=8.93$ & .001 \\
\hline Set sizes 5 vs. 7 & $t(99)=4.47$ & .001 \\
\hline \multicolumn{3}{|l|}{ Main effect of motion } \\
\hline expansion vs. contraction & $t(22)=3.60$ & .002 \\
\hline expansion vs. without motion & $t(22)=3.21$ & .004 \\
\hline contraction vs. without motion & $t(22)=6.81$ & .001 \\
\hline \multicolumn{3}{|l|}{ Main effect of set size } \\
\hline Set sizes 1 vs. 5 & $t(33)=10.75$ & .001 \\
\hline Set sizes 1 vs. 7 & $t(33)=18.80$ & .001 \\
\hline Set sizes 3 vs. 5 & $t(33)=8.90$ & .001 \\
\hline Set sizes 3 vs. 7 & $t(33)=16.96$ & .001 \\
\hline
\end{tabular}

\section{SUPPLEMENTARY TABLE 2.}

Experiment 1B, Results of Post Hoc Tests

\begin{tabular}{lcc}
\hline & $\boldsymbol{t}$ & $\boldsymbol{p}$ \\
\hline Main effect of set size & $t(18)=8.85$ & .001 \\
Set sizes 1 vs. 5 & $t(18)=13.32$ & .001 \\
Set sizes 1 vs. 7 & $t(18)=7.33$ & .001 \\
Set sizes 3 vs. 5 & $t(18)=11.80$ & .001 \\
Set sizes 3 vs. 7 & $t(18)=4.47$ & .001 \\
Set sizes 5 vs. 7 & & \\
Simple main effect of set size & & \\
Expansion & $t(36)=5.65$ & .001 \\
Set sizes 1 vs. 5 & $t(36)=9.37$ & .001 \\
Set sizes 1 vs. 7 & $t(36)=4.31$ & .001 \\
Set sizes 3 vs. 5 & $t(36)=8.03$ & .001 \\
Set sizes 3 vs. 7 & $t(36)=3.72$ & .001 \\
Set sizes 5 vs. 7 & & \\
Contraction & $t(36)=9.07$ & .001 \\
Set sizes 1 vs. 5 & $t(36)=12.79$ & .001 \\
Set sizes 1 vs. 7 & $t(36)=7.88$ & .001 \\
Set sizes 3 vs. 5 & $t(36)=11.60$ & .001 \\
Set sizes 3 vs. 7 & $t(36)=3.72$ & .001 \\
\hline Set sizes 5 vs. 7 & &
\end{tabular}

\begin{tabular}{lcc}
\hline $\begin{array}{l}\text { SUPPLEMENTARY TABLE } 3 . \\
\text { Experiment 2A, Results of Post Hoc Tests }\end{array}$ & \\
\hline & $\boldsymbol{t}$ & \\
\hline Main effect of set size & & \\
Set sizes 1 vs. 5 & $t(24)=7.48$ & .001 \\
Set sizes 1 vs. 7 & $t(24)=11.79$ & .001 \\
Set sizes 3 vs. 5 & $t(24)=6.32$ & .001 \\
Set sizes 3 vs. 7 & $t(24)=10.63$ & .001 \\
Set sizes 5 vs. 7 & $t(24)=4.31$ & .001 \\
\hline
\end{tabular}

\section{SUPPLEMENTARY TABLE 4.}

Experiment 4, Results of Post Hoc Tests

\begin{tabular}{lcc}
\hline & $\boldsymbol{t}$ & $\boldsymbol{p}$ \\
\hline Main effect of set size & & \\
Set sizes 3 vs. 5 & $t(20)=8.58$ & .001 \\
Set sizes 3 vs. 7 & $t(20)=17.76$ & .001 \\
$\quad$ Set sizes 5 vs. 7 & $t(20)=9.17$ & .001 \\
Simple main effect of set size & & \\
Expansion & & \\
Set sizes 3 vs. 5 & $t(40)=6.12$ & .001 \\
Set sizes 3 vs. 7 & $t(40)=13.10$ & .001 \\
Set sizes 5 vs. 7 & $t(40)=7$ & .001 \\
Contraction & & \\
Set sizes 3 vs. 5 & $t(40)=8.06$ & .001 \\
Set sizes 3 vs. 7 & $t(40)=16.24$ & .001 \\
Set sizes 5 vs. 7 & $t(40)=8.18$ & .001 \\
\hline
\end{tabular}

